\title{
Severe Events Related to Use of Stents in Bariatric Surgical Complications
}

\author{
Angelo lossa, MD, Fausto Fiocca, MD, Mario Corona, MD, Giuseppe Cavallaro, MD, PhD, \\ Fabrizio Cereatti, MD, Mario Rizzello, MD, PhD, Gianfranco Silecchia, MD, PhD \\ Department of Medical Surgical Sciences and Biotechnologies, Division of General Surgery and Bariatric Center of \\ Excellence "ICOT," Sapienza University of Rome, Rome, Italy (Drs. lossa, Cavallaro, Cereatti, Rizzello, Silecchia). \\ Department of Surgical Sciences and Organ Transplantation-P. Stefanini, Sapienza University of Rome, Rome, Italy (Dr. Fiocca). \\ Vascular and Interventional Unit, Department of Radiological Sciences, Sapienza University of Rome, Rome, Italy (Dr. Corona).
}

\begin{abstract}
Introduction: Endoscopic self-expandable stents are emerging as safe and effective options for the treatment of bariatric surgery complications. Our aim is to report 3 cases of severe complications after self-expandable stent implantation.

Case Description: A retrospective database analysis showed 14 major complications over the past 400 bariatric procedures (3.5\%); 8 of them were managed conservatively. Three cases of unusual severe complications after self-expandable stent implantation were observed. In case 1 (leak after resleeving), the self-expandable stent migrated twice and caused an esophageal stenosis, which was treated by endoscopic dilation. Three months later, the stenosis recurred and the patient was a candidate for distal esophagectomy. In case 2 (gastrojejunal stricture after Roux-en-Y gastric bypass), the endoscopic dilation was complicated by perforation and treated with a self-expandable stent. The patient required an emergency laparoscopy to remove the stent that had migrated into the ileum. After 1 month, gastrojejunal stenosis recurred and the patient underwent laparoscopic revision of the anastomosis. In case 3 (leak after vertical banded gastroplasty [VBG]-Roux-en-Y gastric bypass conversion), the self-expandable stent migrated twice and caused an esophageal-pleural fistula managed with a new stent.
\end{abstract}

Discussion: Bariatric surgeons have to balance the possible advantages of self-expandable stents on a case-by-case basis. Complications of endoscopic stents can be life-threatening and are underestimated and under-reported in the literature.

Key Words: Bariatric surgery complications, Endoscopic management, Stent complication.

Citation Iossa A, Fiocca F, Corona M, Cavallaro G, Cereatti F, Rizzello M, Silecchia G. Severe events related to use of stents in case of bariatric complications. CRSLS e2014.002234. DOI: $10.4293 /$ CRSLS.2014.002234.

Copyright (c) 2014 SLS This is an open-access article distributed under the terms of the Creative Commons Attribution-Noncommercial-ShareAlike 3.0 Unported license, which permits unrestricted noncommercial use, distribution, and reproduction in any medium, provided the original author and source are credited.

Address correspondence to: Angelo Iossa, MD, Department of Medical Surgical Sciences and Biotechnologies, and Division of General Surgery "ICOT”, La Sapienza, University of Rome, Via Faggiana 1668, 04100 Latina (LT), Rome, Italy. Telephone: +39-0773-6513304, Fax: +39-0773-6513333, E-mail: angelo.iossa@gmail.com

\section{INTRODUCTION}

Bariatric surgery is the only effective treatment that results in clinically significant and sustainable weight loss and comorbidity remission. Sleeve gastrectomy (SG) and Roux-en-Y gastric bypass (RYGB) are the most commonly performed laparoscopic bariatric procedures. ${ }^{1}$ The most frequent complications of both procedures are anastomotic or staple-line leaks and stenosis. Two systematic reviews showed that the reported incidence of leaks after SG is 2.4\% and reaches 13\% with revision surgery. 2,3 The leaks occur more frequently in the proximal third of the staple line near the gastroesophageal junction (85\%-89\%), ${ }^{1}$ with an associated mortality rate of $0.1 \%{ }^{4}$ The incidence of anastomotic leaks after RYGB has ranged from $0.3 \%$ to $8.3 \%,{ }^{5}$ whereas the gastrojejunal anastomotic stricture rate has been reported in a wide range from $3 \%$ to $22 \% .^{5}$ Management of upper gastrointestinal anastomotic complications is complex and challenging especially in the morbidly obese population. In up to one third of patients with fistulas, conservative management fails and the patients require surgical treatment ${ }^{6,7}$ despite the fact that surgical management has been associated with high morbidity and mortality rates. ${ }^{8,9}$

Treatment of upper gastrointestinal complications, such as leaks and strictures, using endoscopic covered self-ex- 
pandable metal stents (SEMSs) is emerging as a safe and effective alternative especially in the case of an early postoperative leak. ${ }^{10-20}$ The benefits of an endoscopic approach in the management of compromised, high-risk morbidly obese patients have been extensively described by several authors. ${ }^{10-20}$ During the past few years, several studies have reported encouraging results, with high success rates of up to $80 \% \cdot{ }^{10,12,13,17-20}$ However, several complications due to endoprosthesis position (mainly dislodgement) have been reported. $9,10,13-19$ The results of the recent literature showed a high failure rate and complications in patients with chronic leaks and therefore recommended earlier implantation of endoscopic stent. 16,20

We retrospectively analyzed a prospective database of $>400$ bariatric operations performed starting in November 2012 in our bariatric center of excellence. The analysis showed 14 major complications (3.5\%): 6 leaks (3 after SG, 1 after primary RYGB, 1 after revision SG, and 1 after revision RYGB); 5 cases of postoperative bleeding; and 3 strictures ( 1 after primary SG and 2 after gastrojejunal anastomosis). Of these complications, 8 ( 5 leaks and 3 cases of bleeding) were managed conservatively. In 3 cases we used endoscopic stenting. Our aim is to report 3 cases of unusually severe adverse events related to endoprosthesis use.

\section{CASE DESCRIPTION}

\section{Case 1}

A 41-year-old man with morbid obesity (body mass index [BMI] of $48 \mathrm{~kg} / \mathrm{m}^{2}$ ) complicated by type 2 diabetes mellitus, hypertension, and severe obstructive sleep apnea syndrome who underwent SG in 2006 was referred to our institution in March 2013 because of weight regain, as well as severe gastroesophageal reflux disease (GERD). The nadir BMI achieved was $31 \mathrm{~kg} / \mathrm{m}^{2}$ two years earlier, with comorbidity resolution. At the first visit, a BMI of $42 \mathrm{~kg} / \mathrm{m}^{2}$ was recorded. Barium swallow and upper gastrointestinal endoscopy showed pouch dilation with evidence of "neofundus." Gastric computed tomography (CT) volumetric analysis showed a total pouch volume of $430 \mathrm{~mL}$ (normal volume $\leq 250 \mathrm{~mL}$ ). After nutritional and psychological assessment, in May 2013, the patient underwent laparoscopic resleeving plus cruroplasty because of an intraoperative finding of a hiatal defect. The patient was discharged on postoperative day 4 after an oral methylene dye test and Gastrografin ${ }^{\circledR}$ (Bayer SPA, Milan, Italy) swallow.
Six days after discharge, the patient was readmitted to a community hospital because of fever and pain in the left upper abdominal quadrant. Blood tests showed leukocytosis (white blood cell count, $20.3 \times 10^{3} / \mu \mathrm{L}$; neutrophils $89 \%)$ and a left pleural effusion on chest radiography. The patient was referred to our center, and a CT scan showed a staple-line leak at the gastroesophageal junction with a left subphrenic abscess $(6.5 \times 5 \mathrm{~cm})$. A conservative management protocol was adopted: total enteral nutrition through a nasojejunal feeding tube, broad-spectrum antibiotic intravenous (IV) therapy, IV proton pump inhibitor (PPI), and percutaneous drainage (CT guided) of the subphrenic abscess. A CT scan after 30 days showed the resolution of the pleural effusion and marked reduction of the abscess with persistence of the leak. An endoscopic retrievable self-expanding metal-covered stent (Megastent, $23 \mathrm{~cm} \times 28 \mathrm{~mm}$; TaeWoong Medical, Seoul, South Korea) was placed under endoscopic and fluoroscopic guidance using moderate sedation and anesthesia support (Figure 1). Six days after the endoscopic placement, the plain radiograph obtained because of acute and persistent vomiting showed distal migration of the stent for about 7

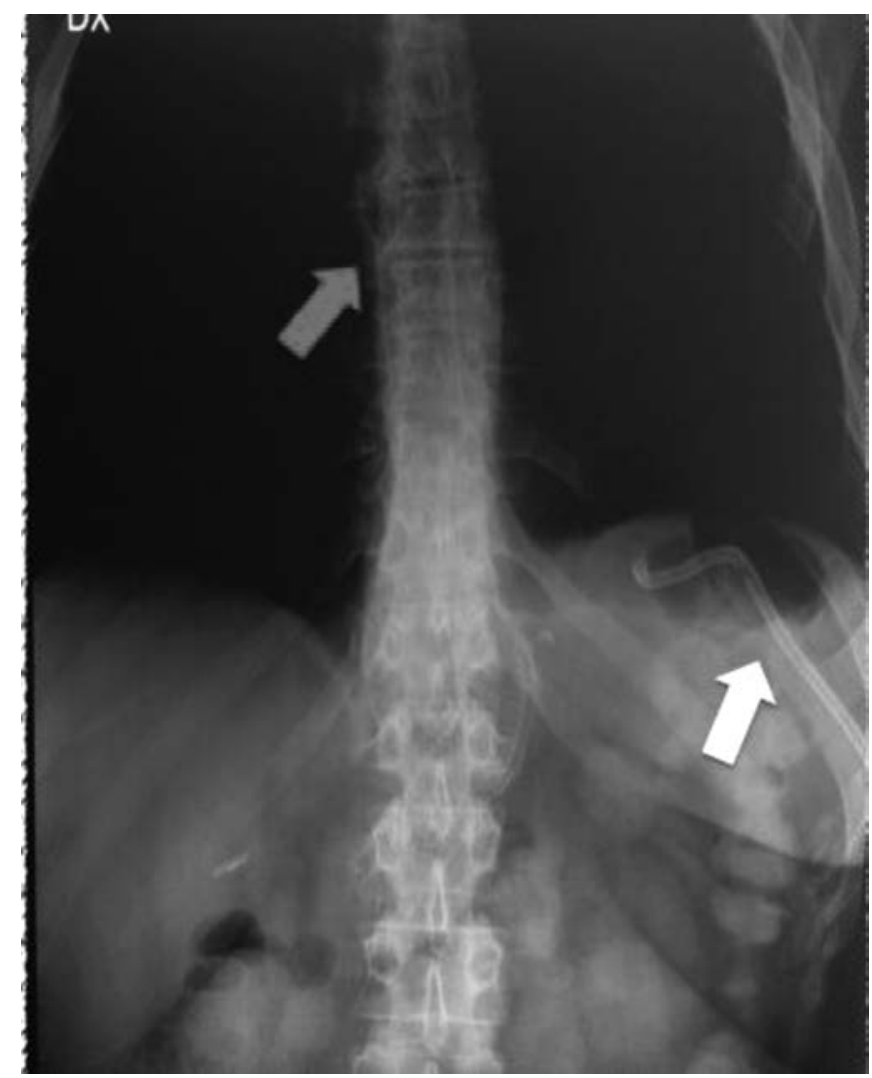

Figure 1. Megastent (red arrow) and abdominal drainage (white arrow) 
$\mathrm{cm}$. The endoprosthesis was repositioned, but unfortunately, after 3 days, it migrated again. The stent was removed, and the patient refused placement of a second stent. After 3 weeks, the patient agreed to undergo endoscopic placement of a second Megastent $(23 \mathrm{~cm} \times 28$ $\mathrm{mm}$ ), which remained in place for 6 weeks. Endoscopy scheduled to remove the stent showed an esophageal stenosis $2 \mathrm{~cm}$ above the endoprosthesis with concomitant esophagitis (possibly due to alkaline) (Figure 2). Because of an inability to overcome the stricture, we proceeded with stenosis dilation with a balloon up to $15 \mathrm{~mm}$ before stent removal. The patient was discharged 103 days after leak diagnosis. He was lost to follow-up. Three months later, a severe stenosis of the distal esophagus was diagnosed at another center and sequential endoscopic dilation failed. The patient subsequently underwent distal esophagectomy and gastrectomy with esophageal-jejunal reconstruction.

\section{Case 2}

A 39-year-old woman with obesity (BMI of $45.7 \mathrm{~kg} / \mathrm{m}^{2}$ ) and a hiatal hernia with severe GERD with class C esophagitis (Los Angeles classification) underwent laparoscopic

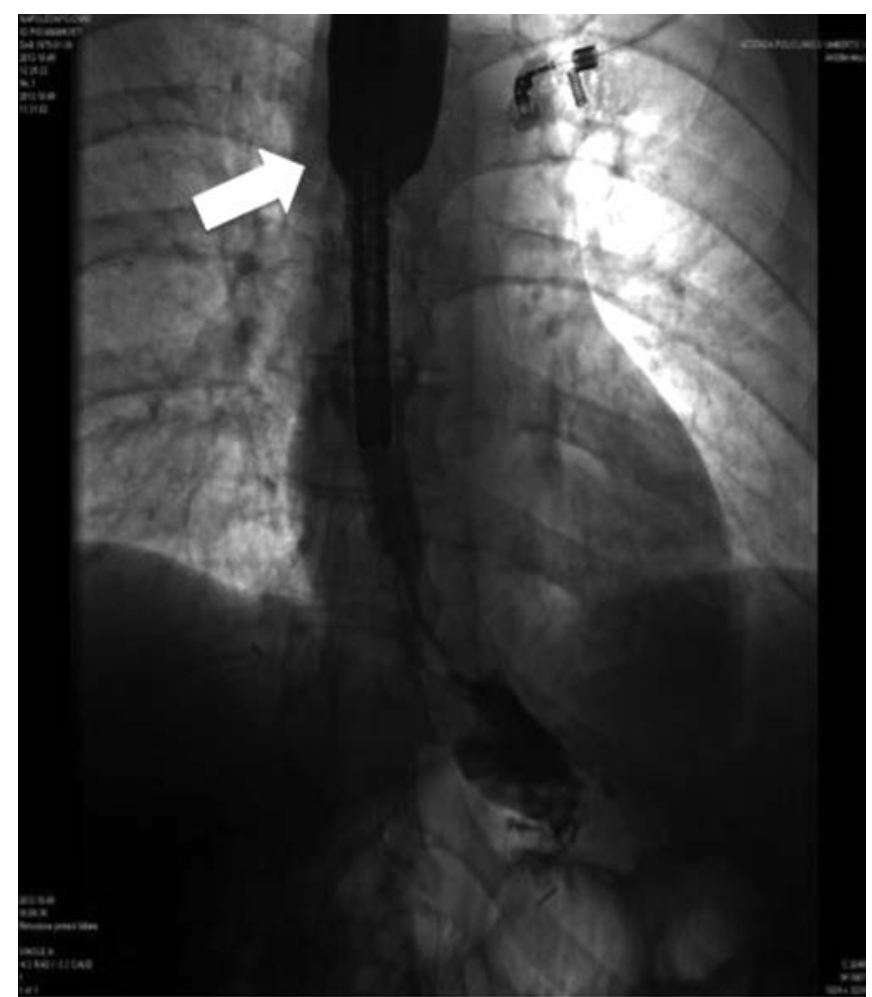

Figure 2. Stenosis at superior limit of stent (arrow) with esophageal dilation. standard RYGB in August 2013. The gastrojejunal anastomosis was constructed with No. 2-0 polyglactin sutures using a totally hand-sewn technique, calibrated with a $36 \mathrm{~F}$ boogie. The patient was discharged on postoperative day 5 after a negative oral methylene dye test finding, Gastrografin swallow, and good tolerance of an oral liquid diet. At 2 weeks postoperatively, patient reported an improvement of GERD symptoms and progression from mushy to solid food intake. Forty-five days after the operation, the patient was readmitted to our institution because of vomiting, dehydration, and dysphagia. On barium swallow, a severe stenosis of the gastrojejunal anastomosis with thread-like passage of barium was diagnosed. Upper gastrointestinal endoscopy confirmed the stenosis with a concomitant marginal ulcer. The patient started a total parenteral nutrition regimen and high-dose IV PPI therapy. After 14 days, repeat endoscopy showed ulcer healing and the first endoscopic balloon dilation to a $10-\mathrm{mm}$ diameter was carried out. The patient restarted an oral diet and was weaned from parenteral nutrition. After 7 days, a second endoscopic balloon dilatation to $15 \mathrm{~mm}$ was planned. Unfortunately, the second dilation was complicated by anastomosis perforation that was treated with a compound ComVi enteral stent $(12 \mathrm{~cm} \times 28 \mathrm{~mm}$; TaeWoong Medical). The patient was discharged after 5 days with an oral liquid diet and PPI therapy.

Eight days after hospital discharge, the patient was readmitted complaining of abdominal pain and vomiting. The plain radiograph showed small bowel air-fluid levels with endoprosthesis migration distally, at about $80 \mathrm{~cm}$ from the ileocecal valve (Figure 3). We proceeded to perform emergency laparoscopy for endoprosthesis removal (Figure 4). The patient was discharged on postoperative day 4 , but the gastrojejunal stenosis recurred and she underwent successful laparoscopic revision of the anastomosis after 5 weeks.

\section{Case 3}

A 57-year-old woman with morbid obesity (BMI of 45 $\mathrm{kg} / \mathrm{m}^{2}$ ), hypertension, severe obstructive sleep apnea syndrome, and asthma presented in 2004 to undergo an open Mason procedure and was referred to our institution in September 2013 for weight regain and GERD. The nadir BMI achieved was $40 \mathrm{~kg} / \mathrm{m}^{2}$ three years earlier, with hypertension resolution. On barium swallow, a gastrogastric fistula was diagnosed, and it was confirmed on endoscopy, with a concomitant hiatal hernia and class $\mathrm{B}$ esophagitis. In November 2013 a laparoscopic conversion to RYGB was performed. A 45-mm blue load gastrointestinal anastomosis linear stapler was used to create a gas- 


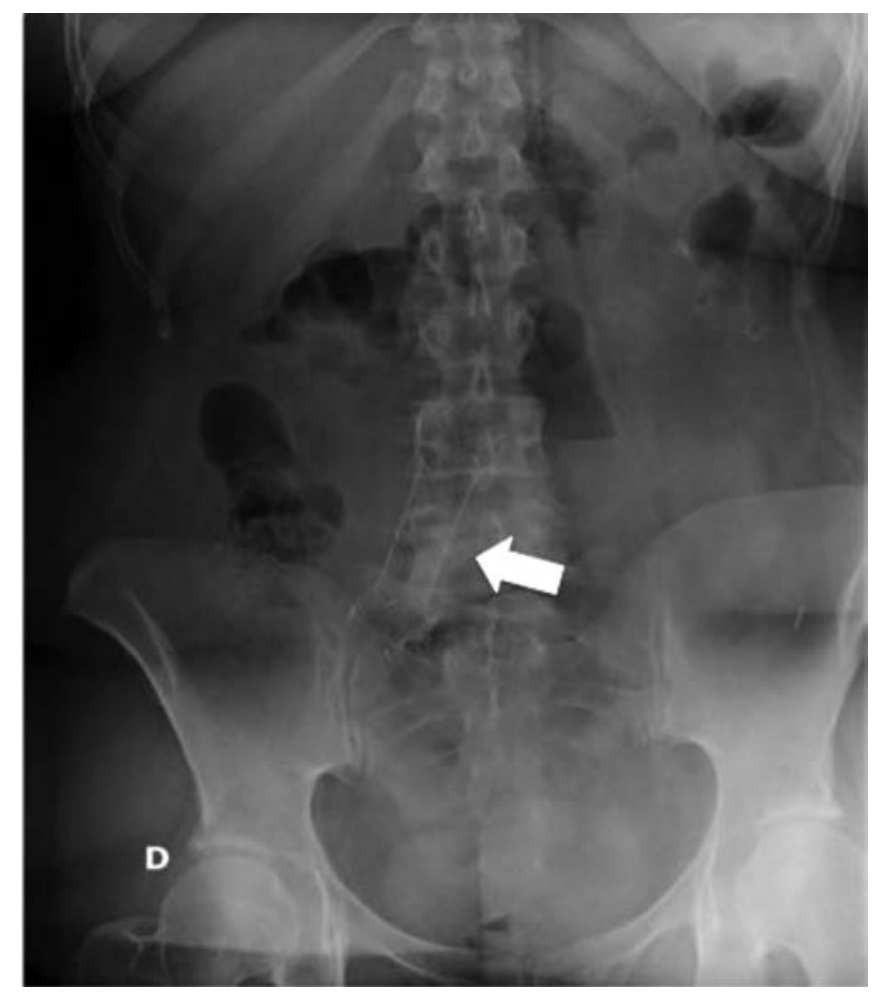

Figure 3. Ileal dislocation of stent (arrow).

trojejunostomy, without using the full load of the linear cartridge in an attempt to fashion an anastomosis that was not longer than $2.5 \mathrm{~cm}$. After 6 days of intensive care unit recovery because of acute respiratory distress, a methylene blue oral test showed a positive finding. A CT scan carried out on the same day showed a gastrojejunostomy leak without evidence of intra-abdominal abscess. After nasojejunal feeding tube insertion, total enteral nutrition was started.

After 40 days of conservative management, a CT scan showed a gastrogastric fistula. Considering the high anesthesiology risk and the intraoperative findings, in January 2013, we proceeded to place a retrievable self-expanding metal stent completely covered by silicone (Megastent, 23 $\mathrm{cm} \times 28 \mathrm{~mm}$ ) under endoscopic and fluoroscopic guidance using moderate sedation. After 3 days, radiographs showed distal migration, and the endoprosthesis was endoscopically replaced without complication. Acute respiratory distress then developed, and the patient was admitted to the intensive care unit. A CT scan showed a conspicuous right pleural effusion with concomitant basal atelectasis and thin pneumothorax requiring placement of 2 chest tubes. After 48 hours, a Gastrografin swallow showed an esophageal and right pleural fistula caused by

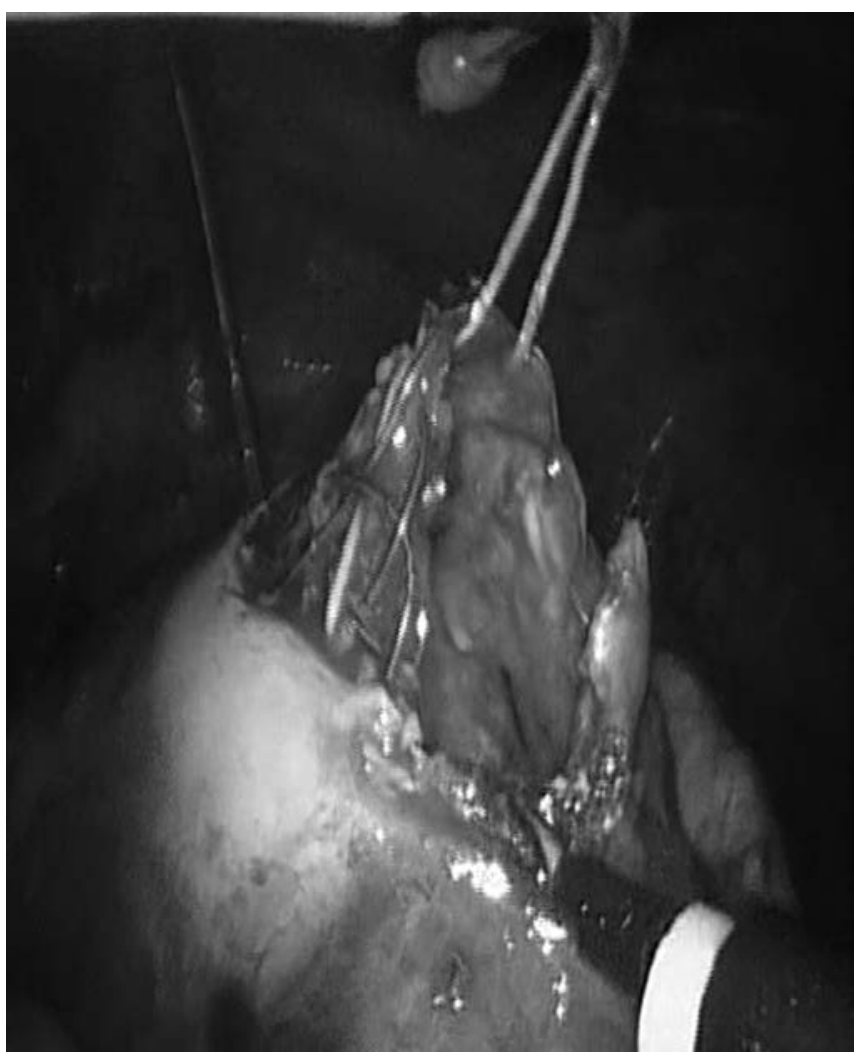

Figure 4. Laparoscopic stent removal.

erosion of the upper stent portion (Figure 5). The stent was removed, and 1 week later, when the patient's general condition improved and sepsis was controlled, a nitinol enteral stent covered in silicone (Niti-S Enteral Colonic Stent, $15 \mathrm{~cm} \times 24 \mathrm{~mm}$; TaeWoong Medical) was placed to isolate the esophageal-pleural fistula. The patient started respiratory rehabilitation, and a weekly stent radiologic control was planned. After 30 days, CT showed pleural effusion resolution and absence of esophageal leak. The residual pulmonary infection was successfully treated by IV antibiotics. The patient was discharged 186 days after leak diagnosis.

\section{DISCUSSION}

Endoscopic stents have been used as a temporary procedure aimed at "bypassing" fluids from the defective area while healing occurs. Prospective studies and guidelines making recommendations on the timing of positioning and removal, type of stent, complications, and cost-effectiveness are lacking. Outcomes after SEMS placement for management of post-bariatric surgery complications are controversial (Table 1). In a meta-analysis involving 67 


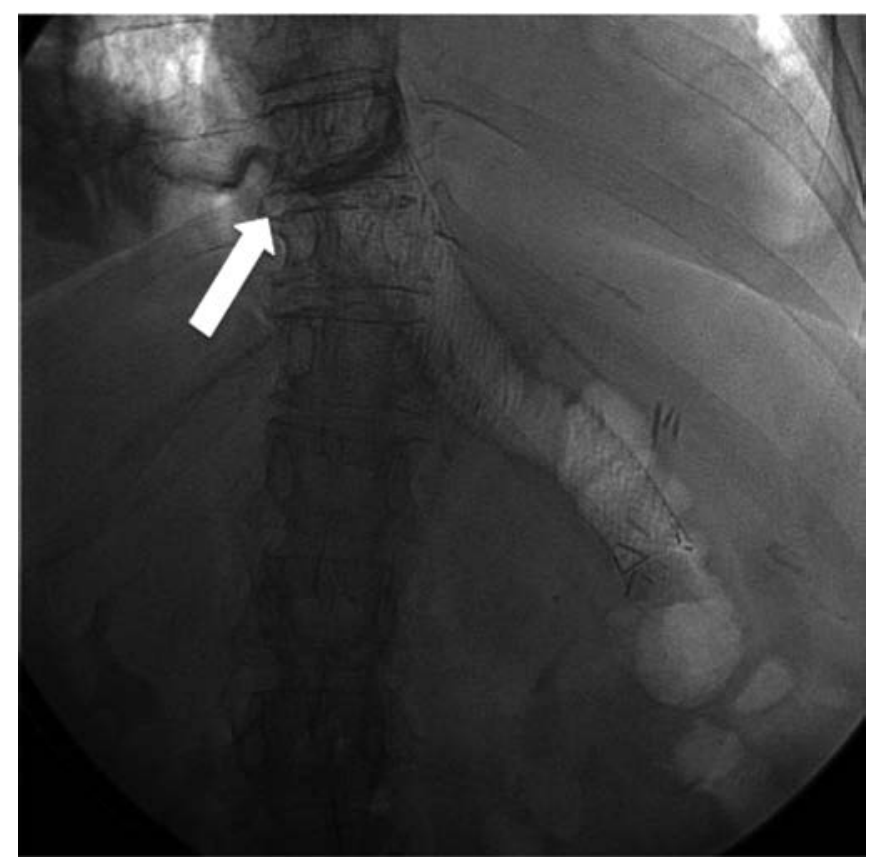

Figure 5. Esophageal and right pleural leak at upper side of stent (arrow).

patients, the pooled proportion of successful closures of anastomotic or staple-line leaks in SEMS cases was 88\% and the stents could be extracted successfully in $92 \%$ of cases. ${ }^{16}$ Stent migration occurred in 17\%. Only 6 of 67 patients required operative revision surgery.

Recently, Donatelli et al, ${ }^{18}$ using uncoated stents in 9 patients affected by acute leaks after SG, reported a success rate of $89 \%$ and migration in $33.3 \%$. In 47 patients, El Mourad et al ${ }^{19}$ reported a success rate of $87.2 \%$, with a complication rate of $25 \%$. Bège et $\mathrm{al}^{1{ }^{17}}$ in a prospective study, showed that the interval between the development or diagnosis of staple-line fistula and SEMS placement influenced outcomes. Early treatment ( $<30$ days) showed an early resolution and required fewer endoscopic sessions. More recently, in a retrospective study involving 21 patients affected by chronic strictures $(\mathrm{n}=16)$ or leaks $(n=5)$, using SEMSs that were partially and fully covered, Puig et al ${ }^{20}$ reported a very low success rate (19\%), with a remarkable migration rate (47\%). Three of these cases required surgical removal. In 14 cases that did not heal with stent management, operative revision was required. The mortality rate was $14.3 \%$. A new method of endoscopic internal drainage plus enteral nutrition (Advanix; Boston Scientific, Boston, Massachusetts), combined with the Over-the-Scope Clip (OTSC; Ovesco Endoscopy AG, Tubingen, Germany), has been described by Donatelli et al, ${ }^{21}$ with a leak healing rate of $95.2 \%$ (20 of 21 cases) after 26 to 180 days. Some authors, on the basis of short case series of nonhomogeneous patients, suggest that stents should remain in place for at least 6 weeks, but there is no consensus. ${ }^{22-24}$ The reported length of time has ranged from 1 to 295 days in the absence of a validated scheme. ${ }^{10-20}$

Although stents offer several advantages in the management of leaks, including the possibility that patients can eat and be managed in the outpatient setting, there are several stent-related problems of note. A structured surveillance program to monitor stent migration is a good policy, based on both patient symptoms and routine imaging at specific intervals. ${ }^{20}$ The type of stent used does not influence the migration index. The shape of the stent, designed primarily for treating esophageal stenosis, is the main reason for high migration rates. New stents designed specifically for bariatric complications could reduce adverse event rates. Recently, several tips and tricks have been proposed to fix the stent (clips, sutures, trans-stent drainage, and so on), but their use is limited to very short case series. ${ }^{25}$ Studies on the long-term efficacy and safety of these techniques are needed. Another potential problem is gastroesophageal reflux when the stent traverses the gastroesophageal junction; indeed, 1 unexpected death that may have been caused by aspiration has been reported. ${ }^{20}$ The cases reported in our study suggest that endoscopic stents handled by a skilled endoscopist in a high-volume bariatric center of excellence can present severe adverse events including migration. The aforementioned complications should be considered in the decision-making process and discussed with patients.

This is the first report of severe esophageal stenosis probably caused by alkaline reflux esophagitis after 6 weeks of stent placement. The stenosis required balloon endoscopic dilation to remove the stent. The stenosis recurred after 3 months, and the endoscopic dilation failed. A severe chronic stenosis of the distal esophagus developed in the patient and was treated by esophageal gastrectomy. Endoscopic dilation of a gastrojejunal anastomotic stenosis after RYGB is the first option, with a reported success rate near $100 \%$, particularly in the case of early stenosis. ${ }^{15,26}$ In our patient the sequential endoscopic dilation caused an anastomotic perforation. The concomitant treatment of the perforation with a stent resulted in distal migration of the stent after a few days, which required laparoscopic removal. The gastrojejunal anastomotic stenosis recurred, and the patient underwent laparoscopic revision of the anastomosis. The third patient was a very complex case in which endoscopic management of a 


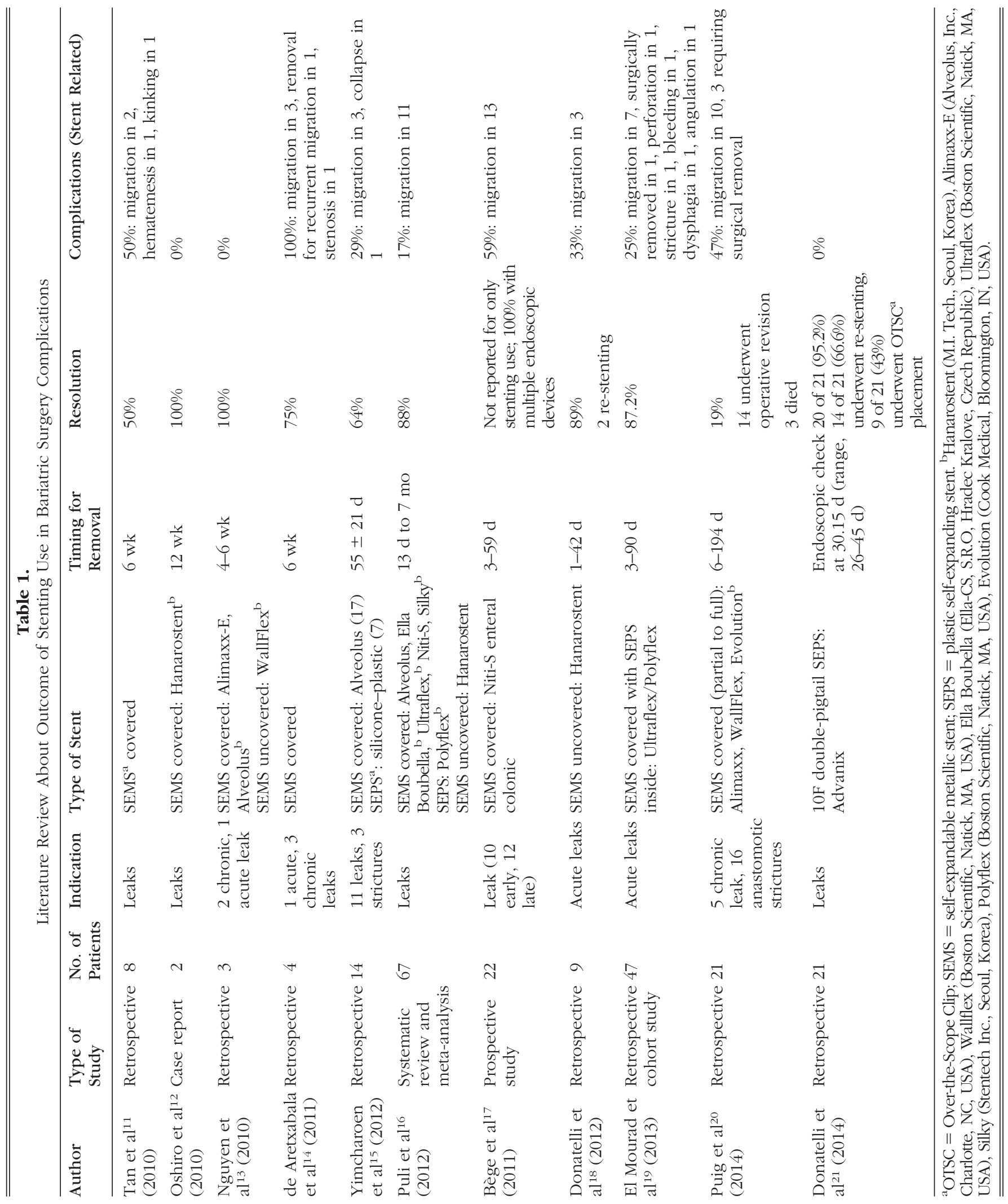


bariatric complication induced severe life-threatening complications. This is the first report of an esophagealpleural fistula caused by a Megastent proximal erosion after a few days of positioning (10 days). The prompt diagnosis of this unusual complication and treatment were crucial in the successful management of this case.

Our management of bariatric complications has changed in terms of patient selection and strategy choice. We consider an SES only in cases of acute leaks (1 week after surgery), we schedule intensive radiologic monitoring (twice a week), and we no longer place an SEMS in cases of leaks after RYGB. New Megastents (longer and larger than those available) specifically designed for bariatric patients with safe and effective antimigration systems are advocated to reduce the high dislodgement rate. The unusual and severe complications reported underline the need for guidelines on the use of stents in the management of post-bariatric surgery complications including follow-up scheduling and treatment of stent-related complications.

\section{CONCLUSIONS}

The high success rate encourages the wide use of large and long coated stents, which offers a chance of nonsurgical management in complex patients, with use as early as possible. ${ }^{19}$ The high percentage of complications in chronic cases ( $>30$ days) strengthens the need for continuous monitoring by a multidisciplinary team in a highvolume bariatric center. We have reported 3 severe unusual complications of endoscopic stents used for the treatment of bariatric complications. Bariatric surgeons have to balance, on a case-by-case basis, the possible advantages versus disadvantages of endoscopic management of stenosis and leaks after laparoscopic bariatric procedures. In the absence of guidelines, each patient should be carefully informed about these possible severe complications, the high failure rate in chronic fistula cases, and possible operative management.

\section{References:}

1. Buchwald H, Oien DM. Metabolic/bariatric surgery worldwide 2011. Obes Surg. 2013;23(4):427-436.

2. Gagner M, Buchwald JN. Comparison of laparoscopic sleeve gastrectomy leak rates in four staple-line reinforcement options: a systematic review. Surg Obes Relat Dis. 2014;10(4):713-723.

3. Clinical Issues Committee of the American Society for Metabolic and Bariatric Surgery. Updated position statement on sleeve gastrectomy as a bariatric procedure. Surg Obes Relat Dis. 2010;6:1-5.
4. Parikh M, Issa R, McCrillis A, et al. Surgical strategies that may decrease leak after laparoscopic sleeve gastrectomy: a systematic review and meta-analysis of 9991 cases. Ann Surg. 2013; 257:231-237.

5. Yimcharoen P, Heneghan HM, Tariq N, et al. Endoscopic stent management of leaks and anastomotic strictures after foregut surgery. Surg Obes Relat Dis. 2011;7(5):628-636.

6. Carrodeguas L, Szomstein S, Soto F, et al. Management of gastrogastric fistulas after divided Roux-en-Y gastric bypass surgery for morbid obesity: analysis of 1,292 consecutive patients and review of literature. Surg Obes Relat Dis. 2005;1:467-474.

7. Gumbs AA, Duffy AJ, Bell RL. Management of gastrogastric fistula after laparoscopic Roux-en-Y gastric bypass. Surg Obes Relat Dis. 2006;2:117-121.

8. Gonzalez R, Sarr MG, Smith CD, et al. Diagnosis and contemporary management of anastomotic leaks after gastric bypass for obesity. J Am Coll Surg. 2007;204:47-55.

9. Madan AK, Martinez JM, Lo Menzo E, et al. Omental reinforcement for intraoperative leak repairs during laparoscopic Roux-en-Y gastric bypass. Am Surg. 2009;75:839-842.

10. Edwards CA, Bui TP, Astudillo JA, et al. Management of anastomotic leaks after Roux-en-Y bypass using self-expanding polyester stents. Surg Obes Relat Dis. 2008;4(5):594-599; discussion 599-600.

11. Tan JT, Kariyawasam S, Wijeratne T, et al. Diagnosis and management of gastric leaks after laparoscopic sleeve gastrectomy for morbid obesity. Obes Surg. 2010;20(4):403-409.

12. Oshiro T, Kasama K, Umezawa A, et al. Successful management of refractory staple line leakage at the esophagogastric junction after a sleeve gastrectomy using the HANAROSTENT. Obes Surg. 2010;20:530-534.

13. Nguyen NT, Nguyen XM, Dholakia C. The use of endoscopic stent in management of leaks after sleeve gastrectomy. Obes Surg. 2010;20:1289-1292.

14. de Aretxabala X, Leon J, Wiedmaier G, et al. Gastric leak after sleeve gastrectomy: analysis of its management. Obes Surg. 2011;21(8):1232-1237.

15. Yimcharoen P, Heneghan H, Chand B, et al. Successful management of gastrojejunal strictures after gastric bypass: is timing important? Surg Obes Relat Dis. 2012;8:151-157.

16. Puli SR, Spofford IS, Thompson CC. Use of self-expandable stents in the treatment of bariatric surgery leaks: a systematic review and meta-analysis. Gastrointest Endosc. 2012;75:287-293.

17. Bège T, Emungania $\mathrm{O}$, Vitton $\mathrm{F}$, et al. An endoscopic strategy for management of anastomotic complications from bariatric surgery: a prospective study. Gastrointest Endosc. 2011;73:238-244.

18. Donatelli G, Dhumane P, Perretta S, et al. Endoscopic placement of fully covered self expanding metal stents for manage- 
ment of post-operative foregut leaks. J Minim Access Surg. 2012; 8(4):118-124.

19. El Mourad H, Himpens J, Verhofstadt J. Stent treatment for fistula after obesity surgery: results in 47 consecutive patients. Surg Endosc. 2013;27(3):808-816.

20. Puig CA, Waked TM, Baron TH, et al. The role of endoscopic stents in the management of chronic anastomotic and staple line leaks and chronic strictures after bariatric surgery. Surg Obes Relat Dis. 2014;10(4):613-617.

21. Donatelli G, Ferretti S, Vergeau BM, et al. Endoscopic internal drainage with enteral nutrition (EDEN) for treatment of leaks following sleeve gastrectomy. Obes Surg. 2014;24(8):1400-1407.

22. Eubanks S, Edwards CA, Fearing NM, et al. Use of endoscopic stents to treat anastomotic complications after bariatric surgery. J Am Coll Surg. 2008;206(5):935-938; discussion 938939.

23. Baltasar A, Serra C, Perez N, et al. Laparoscopic sleeve gastrectomy. A multipurpose bariatric operation. Obes Surg. 2005;15:1124-1128.

24. Baltasar A, Bou R, Bengochea M, et al. Mil operaciones bariatricas. Cir Esp. 2006;79:349-353.

25. Rieder E, Dunst CM, Martinec DV, et al. Endoscopic suture fixation of gastrointestinal stents: proof of biomechanical principles and early clinical experience. Endoscopy. 2012;44:11211126.

26. Ukleja A, Afonso BB, Pimentel R, et al. Outcome of endoscopic balloon dilation of strictures after laparoscopic gastric bypass. Surg Endosc. 2008;22(8):1746-1750. 